\title{
Estudio de procedimientos para mejorar el sistema de calidad en el hospital de especialidades "José Carrasco Arteaga" del Instituto Ecuatoriano de Seguridad Social Cuenca - Azuay
}

Study of Procedures to Improve the Quality System in the Hospital of Specialties "José Carrasco Arteaga" of the Ecuadorian Institute of Social Security Cuenca - Azuay

Eulalia Catalina Torres Dávila1, María Fernanda López Espinoza², Verónica Paulina Cáceres Manzano ${ }^{3}$, \& Oscar David Espín Maldonado ${ }^{4}$

Recibido: 10-02-2019 / Revisado: 15-02-209 /Aceptado: 04-03-2019/ Publicado: 27-04-2019

\begin{abstract}
.
DOI: https://doi.org/10.33262/cienciadigital.v3i2.1.430

Quality in health care is the property or set of care to contribute to the patient in a comprehensive service for good living. There are several components quality of care such as good treatment, good practice, patient identification, maintenance of equipment, use of abbreviations, identification and management of medicines, among others. José Carrasco Arteaga Specialty Hospital maintains a continuous improvement for patient care
\end{abstract}

For the quality system improvement in Specialty Hospital José Carrasco Arteaga Instituto de Seguridad Social Cuenca-Azuay different procedures will be raised which is part of the reform in the patient safety plan.

The new guidelines of the Patient Safety Plan approved in 2018, in its third measurement taken according to quarterly reports of patient safety of the Hospital gave as results that $63 \%$ of safe practices exceed $70 \%$ of the fulfillment of the evaluated services

\footnotetext{
${ }^{1}$ Médico, Médico en Funciones Hospitalarias, Médico Auditor en Facturación. Hospital de Especialidades José Carrasco Arteaga. Cuenca Ecuador.

${ }^{2}$ Médico, Médico Residente en la Unidad Médica Móvil SOLCA. Cuenca Ecuador.

${ }^{3}$ Licenciada en Laboratorio Clínico e Histopatología, Abogada, Master en Seguridad Clínica del Paciente y Calidad Asistencial.

Universidad Nacional de Chimborazo. Riobamba Chimborazo.

${ }^{4}$ Ingeniero, Coordinador General de Control de Calidad. Hospital de Especialidades José Carrasco Arteaga Cuenca Ecuador
} 
The following conclusion of the evaluated services can be obtained: the minimum acceptable value of the fulfillment of required organizational practices reflects an increase with respect to the performance of the services of higher level and service of lower compliance score.

Keywords: Study, Procedures, Quality System, Specialty Hospital

\section{Resumen.}

La calidad en la atención de salud es la propiedad o conjunto de atenciones para contribuir al paciente en un servicio integral para el Buen Vivir. Como parte de la calidad de atención tenemos varios componentes como el buen trato, la buena práctica, la identificación del paciente, el mantenimiento de equipos, la utilización de abreviaturas, identificación y manejo de medicamentos, entre otros. En el Hospital de Especialidades "José Carrasco Arteaga", se mantiene un continuo mejoramiento para la atención del paciente tomando en cuenta las seis áreas analizadas.

Para la mejora del sistema calidad en Hospital de Especialidades "José Carrasco Arteaga“, Instituto de Seguridad Social Cuenca- Azuay vamos a plantear diferentes procedimientos los cuales son parte de la reforma en el plan de Seguridad del Paciente; por medio de un análisis aleatorio de varias especialidades.

Los nuevos lineamientos del Plan de Seguridad del Paciente aprobado en el 2018, en su tercera medición tomada según los informes trimestrales de seguridad del Paciente del Hospital de Especialidades "José Carrasco Arteaga", nos da como resultados que el 63\% de Pràcticas Seguras superan el 70\% de cumplimieto en los servicios evaluados, sobrepasando la línea de base para un mejor desarrollo.

Como conclusión de los servicios evaluados, tanto en el valor mínimo aceptable del cumplimiento de Prácticas Organizacionales requeridas refleja un incremento con respecto al desempeño de los servicios de mayor nivel y servicio de menor puntaje de cumplimiento.

Palabras claves: Estudio, Procedimientos, Sistema de Calidad, Hospital de Especialidades

\section{Introducción.}

En la calidad de atención tenemos varios componentes como el buen trato, la buena práctica, la identificación del paciente, el mantenimiento de equipos, la utilización de abreviaturas, identificación y manejo de medicamentos, entre otros.

La Organización Mundial de la Salud define la calidad asistencial de la siguiente forma (1): "Una atención sanitaria de alta calidad es la que identifica las necesidades de salud educativas, preventivas, protectoras y de mantenimiento de los individuos o de la población de una forma total y precisa, y destina los recursos necesarios (humanos y de 
otros tipos) a estas necesidades de manera oportuna y tan efectiva como el resultado actual del conocimiento lo permite". (1)

Donabedian (1984) define calidad como los logros de los mayores beneficios posibles de la atención médica, con los menores riesgos para el paciente. (2) (3) (12)

Calidad asistencial según la Real Academia Española define calidad como la "propiedad o conjunto de propiedades inherentes a una cosa que permiten apreciarla como igual, mejor o peor que las restantes de su misma especie. (3).

Los atributos o propiedades del proceso de la prestación de atención y otros, como metas u objetivos de ese proceso de Lee y Jones. Los criterios de calidad no son nada más que juicios de valor que se aplican a distintos aspectos, propiedades, componentes o alcances de un proceso denominado atención médica.

La calidad asistencial alrededor del tiempo ha sido tratada desde un inicio por diferentes autores en algunas culturas clásicas en medicina: Asiria, Egipto, China, Grecia, y Roma, encontrándose evidencia en ciertos documentos conservados (papiros egipcios, códigos hipocráticos, documentos chinos, Código de Hammurabi) tratan de materias que podrían incluirse en el campo de la calidad asistencial. Hay varios exponentes de calidad asistencial en la edad Moderna como Sir William Petty (1623-1687) da el enunciado de la necesidad de medir el producto hospitalario, comparando la frecuencia de mortalidad entre hospitales. William Farr (1807-1883) desarrolló un sistema uniforme de clasificación de enfermedades, que facilitó el desarrollo de las estadísticas vitales, Marc D’Espine, de Suiza y Jacques Bertillon, de Francia. Trabajaron juntos para conseguir una clasificación de causas de muerte que se adoptara universalmente. Abraham Flexner en 1910 nos habla de la importancia de autorizar o certificar los proveedores en la atención sanitaria, siempre considerando la comorbilidad. (4)

En los años 70 aumentó la preocupación e incrementó del gasto sanitario en un contexto de crisis económica; en 1972 se formó un sistema de revisión por iguales el objetivo era asegurar que los servicios ofrecidos eran necesarios desde el punto de vista médico.

En 1980 la Organización Mundial de Salud, dentro de la estrategia "Salud para todos en el año 2000", en el objetivo 31, dice: "De aqui a 1990 todos los Estados miembros deberán haber creado unas comisiones eficaces que aseguren la calidad de las atenciones a los enfermos en el marco de sus sistemas de prestaciones sanitarias. Se podrá atender este objetivo si se establecen métodos de vigilancia continua y sistemática para determinar la calidad de los servicios prestados a los enfermos, convirtiendo las actividades de evaluación y control en una preocupación constante de las actividades habituales de los profesionales sanitarios y finalmente, impartiendo a todo el personal sanitario una formación que asegure y amplie sus conocimientos". En 1982, el PSRO fue sustituido por el Peer Review Program (PRO), se centraba en estudiar la necesidad médica, la calidad de la asistencia, y la adecuación de la utilización, el PRO podía recomendar sanciones, no sólo contra hospitales, sino también contra enfermeras y médicos. (4)

En los años 90 se puso más énfasis y comenzó a tener fuerza en el mundo en 1994, los principales medidores de los estándares de calidad a los ciudadanos y la entera responsabilidad a los profesionales de la salud. Joint Commission (JCAHO), es el grado del servicio de atención al paciente. En 1988 la OTA (Office of Technology 
Assessment), calidad asistencial es el nivel del proceso de atención que aumenta la probabilidad de resultados deseados por el enfermo y reduce la probabilidad de efectos adversos dado el estado de conocimiento técnico reduciendo la relación riesgo/beneficio de cualquier actividad médica y hacer las cosas correctamente y hacer lo correcto; los profesionales en la salud aportan una perspectiva individual de la calidad en su práctica clínica, haciendo lo correcto de la forma más correcta. Los pacientes aportan una perspectiva individual, al exigir competencia técnica, accesibilidad y comprensión. Los administradores y las organizaciones sanitarias, buscan un equilibrio entre el rendimiento técnico, la satisfacción de los pacientes y el gasto económico asumible. Ángel Otero propone una definición: "la provisión de servicios sanitarios adecuados al conocimiento, accesibles y equitativos, con un nivel profesional óptimo, y con el mínimo riesgo para los pacientes, teniendo en cuenta los recursos disponibles y logrando la satisfacción del usuario”. (4)

La calidad asistencial es una disciplina que ha adquirido una enorme relevancia en la atención sanitaria actual. Los pacientes cada vez son más expertos y responsables; los profesionales, más capaces y mejor formados; y la tecnología médica, más avanzada y resolutiva. (4)

Los indicadores para la calidad asistencial son tres: a) Los Indicadores de estructura, todos los atributos materiales y organizacionales relativamente estables, así como los recursos humanos y financieros disponibles en los sitios en los que se proporciona atención. b) Los Indicadores de proceso. Se refieren a lo que los médicos y proveedores son capaces de hacer por los pacientes, la interrelación con las actividades de apoyo diagnosticadas además de las actitudes, habilidades, destreza y técnica con que se llevan a cabo. c) Los indicadores de resultado. Referidos a la variación de los niveles de salud y a la satisfacción del paciente por la atención recibida, la cual le otorga una importancia mayor por la necesidad de satisfacer las expectativas del usuario mediante un servicio de mayor calidad. (5)

La calidad asistencial es el resultado de tres componentes a) El Componente científicotécnico, la ciencia y la tecnología médica y alcanzar el máximo beneficio con el mínimo riesgo para el paciente. b) Componente interpersonal, las relaciones humanas que deben seguir las normas y valores sociales como la ética profesional y las expectativas del paciente.

C) Componente de confort, desarrolla la asistencia: accesibilidad, condiciones ambientales, instalaciones, hostelería. (5)(9)

El componente científico-técnico se da la mayoría de las veces por supuesto, el nivel de eficacia de la medicina. Los componentes interpersonales y de confort forman parte de lo que entendemos como calidad percibida, y son los más valorados por el paciente y su familia. (5)

Las Prácticas Seguras o Seguridad del Paciente (SP), es el intento consciente de evitar lesiones al paciente causado por la asistencia, es un componente esencial de la Calidad Asistencial y la condición previa para realizar cualquier actividad clínica. Se asientan en dos líneas de pensamiento relacionadas: la primera la teoría del error de Reason y la segunda la cultura de seguridad, conjunto de valores y normas comunes a los individuos, modelo mental compartido que posiciona la seguridad. (6) 
En la calidad asistencial tenemos diferentes protocolos: la identificación del paciente; la pulsera de Identificación; en la hospitalización, es la pernoctación de un paciente en una cama individual en cuartos compartidos y aislados censables del hospital, para su atención de una patología; los datos inequívocos, son aquellos que corresponden a un solo paciente, que no pueden duplicarse, como el número de cédula de ciudadanía, dos nombres y dos apellidos, fecha de nacimiento; en los casos de los recién nacidos se utiliza las pulseras identificativas de la madre del niño; la verificación cruzada, la lista de chequeo, en los diferentes servicios previo durante y posterior a los procedimientos; la identificación con tarjeta de cabecera pie de cama o en la habitación. (6)

El mantenimiento de los equipos es un procedimiento que se realiza de forma planificada con el objetivo de minimizar los fallos que puedan tener los equipos y optimizar su calibración, procesos se incluye limpieza, calibración, ajustes, reemplazo de partes vulneradas para garantizar la seguridad del paciente al usar dicho equipo. Las ventajas del mantenimiento preventivo o prevención de fallas en los equipos o instalaciones, son la reducción de reemplazos de equipos durante su vida útil. La calibración de los equipos consiste en comparar los resultados obtenidos producto del proceso realizado con los patrones o estándares internacionales o normados, actividad que se hace a través de equipos, instrumentos, patrones o estándares. Las pruebas de operatividad, las que consisten en efectuar inspecciones visuales integrales y del funcionamiento, siguiendo normas y procedimientos emitido por institutos, organismos, o asociaciones dedicados a la reglamentación de la construcción y calidad de los equipos médicos con el fin de verificar la eficiencia y seguridad de estos. Los estándares de calidad y funcionamiento son dados por los mismos fabricantes. (6)

Otro elemento importante para una práctica segura en la calidad de atención al paciente es el control de abreviaturas peligrosas las cuales son normatizadas según los acuerdos ministeriales $\mathrm{N}^{\circ}$. 00001124, El Ministerio de Salud Pública, considerando: Que la Constitución de la República del Ecuador Acuerda: Capítulo I:

Art. 1.- El objetivo del presente instructivo es regular y controlar la prescripción y dispensación de los medicamentos y dispositivos médicos en las unidades de salud del Ministerio de Salud Pública.

Art. 2.- La receta médica es un documento asistencial y de control, que permite la comunicación entre el prescriptor, dispensador y paciente, constituye también el documento de control administrativo "Art. 361.- cuando se requiera".

Art. 3.- Están autorizados para prescribir los profesionales: Médicos, Odontólogos y Obstetrices, estrictamente los medicamentos de su especialidad, respetando los niveles de prescripción conforme lo señala el cuadro nacional de medicamentos básicos vigente. (7)

Las Abreviaturas peligrosas son aquellas abreviaturas que tienen significados iguales o tienen similitud entre un medicamento y otro los cuales pueden llevar a confusión. Las abreviaturas permitidas, en la historia clínica, prescripciones, formularios o notas siempre deben ser parte del listado legal como: MC: motivo de consulta, EA: enfermedad actual, DX: diagnóstico, QD: cada día, QUID: cuatro veces al día.

El control y cumplimiento se debe dar en todas la instituciones contando con el comité de mejoramiento continuo de la calidad, el mismo se encargará del manejo de los 
medicamentos de alto riesgo: medios de contraste, inotrópicos, adrenérgicos, anestésicos generales inhalados, antiagregantes plaquetarios, antiarrítmicos, anticoagulantes orales, antidiabéticos orales, bloqueantes neuromusculares, citostáticos, parenterales y orales heparina y otros anticoagulantes parenterales, insulinas IV y subcutáneas, medicamentos para sedación moderada IV, medicamentos orales para sedación moderada en niños, medicamentos que tienen presentación convencional y en liposomas, nutrición parenteral, opiáceos IV, transdérmicos y orales, trombolíticos. Comienza desde la identificación correcta del medicamento, verificando la caducidad y condiciones de almacenamiento. Se obtienen de la farmacia con receta especial y debe ser entregados al personal médico o de enfermería para su transporte y almacenamiento. La comunicación en la práctica médica a diario encontramos una diversidad de criterios, la falta de algunos protocolos de tratamiento e interpretación diferenciada de cada situación. Muchas veces este problema y es reflejado en el despacho y custodia de los medicamentos de alto riesgo. Se debe explicar en forma clara y sencilla el tratamiento, información escrita al alta. La conciliación de los medicamentos es el proceso formal para obtener una lista completa de la medicación del paciente previo al ingreso, compararla con la que se le ha prescrito en el centro sanitario, en los traslados y a la alta médica. (6)

Considera «discrepancia» cualquier diferencia entre la medicación domiciliaria crónica que el paciente tomaba previamente y la medicación prescrita en el hospital. La adaptación a la Guía Fármaco-terapéutica del Hospital, es un instrumento útil y necesario para gestionar el uso racional de los medicamentos en los hospitales; está guía es basada en el Cuadro Básico de Medicamentos del Ministerio de Salud Pública. Los momentos de conciliación de los medicamentos son en la sala de la emergencia, al ingreso, en la estancia de la unidad y al alta. (6)

Para las prácticas seguras en el hospital, se debe tomar en cuenta las siguientes reglas: administración de medicamentos, (los cuales son sustancias que se administran para el diagnóstico, la curación, el tratamiento, la mitigación del dolor, o la prevención de la enfermedad). Kardex de enfermería. Vía intradérmica, vía subcutánea, vía parenteral; posición fowler del paciente siempre en una posición cómoda para administración de medicamentos, se debe tomar en cuenta las reacciones adversas que den pueden tener estos; para una buena atención al paciente se debe lavar las manos previamente, verificar la identificación del paciente, de los medicamentos sus dosis y vía correcta según la prescripción del tratante, posterior a la administración se debe registrar en el expediente clínico la hora, vía y responsable de la colocación de dicho medicamento. (6)

El Hospital Especialidades “José Carrasco“" se inauguró el 3 de noviembre del 2000, en la actualidad ha incrementado y mejorado su servicio en beneficio de los afiliados y pacientes de la Red Pública Integral de Salud contribuyendo el buen vivir; el Hospital ha evolucionado en estos años de 300 camas desde la inauguración en la actualidad existen 380 camas; ha mejorado en tecnología para el servicio de sus pacientes; además la Institución a duplicado el número de consultorios, especialidades, subespecialidades y siendo parte de casas de salud donde se realizan trasplante de cornea, renal y proximamente trasplante hepático, prestando nuestros servicios siempre a beneficio de la ciudadanía. (8) 
La calidad en la atención en el Hospital de Especialidades "José Carrasco Arteaga" se reformula y aprueba en julio del 2018 con el Plan de Seguridad del Paciente con nuevos lineamientos a seguir comunicando primero a nivel interno, además de diseñar un instrumento de trabajo; establecer líneas de base; posterior a eso se sociabilizó, analizó los resultados y se estableció planes de acción para mejora dando también seguimientos; actualmente se encuentra en la tercera medición (7)

\section{Metodologia.}

Para la mejora del sistema calidad en Hospital de especialidades José Carrasco Arteaga Instituto de Seguridad Social Cuenca- Azuay vamos a plantear diferentes procedimientos los cuales son parte de la reforma en el plan de Seguridad del Paciente.

En el presente estudio observacional descriptivo, retrospectivo y aleatorio que se llevó a cabo en las áreas de hospitalización, servicios de transfusión, consulta externa, laboratorio clínico, anatomía patológica oftalmología y central de esterilización.

Los instrumentos que fueron diseñados en base a los requisitos de las prácticas organizacionales requeridas de los estándares de la Acreditación Internacional de Canadá Nivel Oro, Platino y Diamante. Una categoría de evaluación en base al Acuerdo Ministerial 5186 - Manual de Manejo de Desechos, se integró una categoría de evalución correspondiente a limpieza en los servicios, evaluación en prácticas seguras, en todos los servicios se evalúa la disponibilidad de los protocolos vigentes.

Cada categoría es evaluada sobre el 100\% tomando en cuenta los aspectos de estructura, procesos y resultados. Además, nos basamos en la tercera medición de las prácticas organizacionales del plan de seguridad del paciente en informes trimestrales.

\section{Resultados.}

Los nuevos lineamientos del Plan de Seguridad del Paciente aprobado en el 2018, en su tercera medición tomada según los informes trimestrales de seguridad del Paciente del Hospital Especialidades "José Carrasco Arteaga" nos da como resultados el $63 \%$ de Practicas Seguras superan el 70\% de cumplimieto en los servicios evaluados y el 10\% al $44 \%$ mejorando en las prácticas organizacionales en la segunda. Tabla $\mathrm{N}^{\mathrm{a}} 1$

Como se puede apreciar e la Tabla $\mathrm{N}^{\mathrm{a}} 1$ de la información tomada en la segunda medición en el mes de julio comparando con el mes de octubre encontramos que se supera la meta establecida (85\%) junio 2018, esto representa una mejora del $14 \%$ con respecto a la medición previa y un $38 \%$ más a la línea base, se registra un nivel de cumplimiento de únicamente del $6 \%$. 
Tabla Na 1 Tomada de Resultados de Evaluación. Plan de Seguridad del Paciente. Tercera Medición. Hospital José Carrasco Arteaga. Octubre 2018. (14)

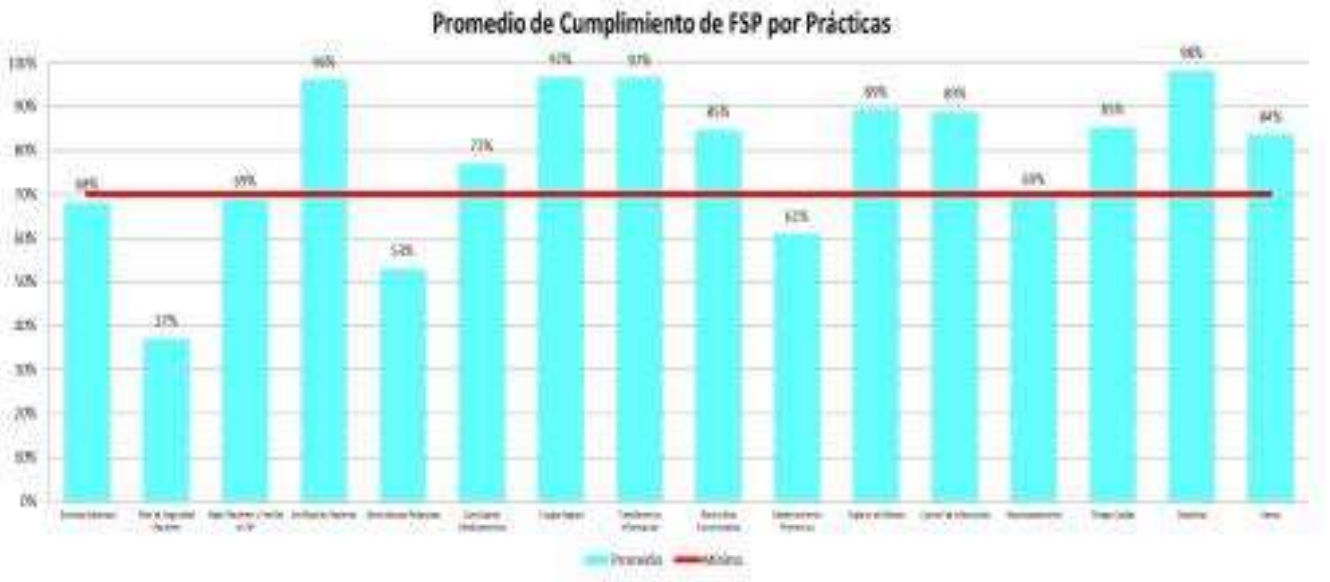

Fuente: Hospital Especialidades "José Carrasco Arteaga"

Elaborado por: autores

Como se puede evidenciar en la Tabla $\mathrm{N}^{\mathrm{a}}$ 2, el $77 \%$ de los servicios evaluados superan el valor mínimo aceptable (70\%) de cumplimiento de las prácticas organizacionales requeridas evaluadas; refleja una mejora con respecto a la medición previa tomada en julio 2018 en la cual se obtuvo el $41 \%$ que superaban el mínimo. El porcentaje de servicios que superan la meta del 85\% se incrementó del 14 al $27 \%$.

En un análisis comparativo de las mediciones en prácticas seguras en los servicios de consulta externa, servicios transfusionales, hospitalización 5to piso, central de esterilización, oftalmología y laboratorio de anatomía patológica podemos observar la evolución de la primera, segunda y tercera medición del plan de seguridad del paciente tomado de los informes trimestrales. 
Tabla Na 2 Tomada de Resultados de Evaluación. Plan de Seguridad del

Paciente. Tercera Medición. Hospital José Carrasco Arteaga. Octubre 2018. (14)

Consulta Externa

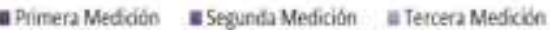
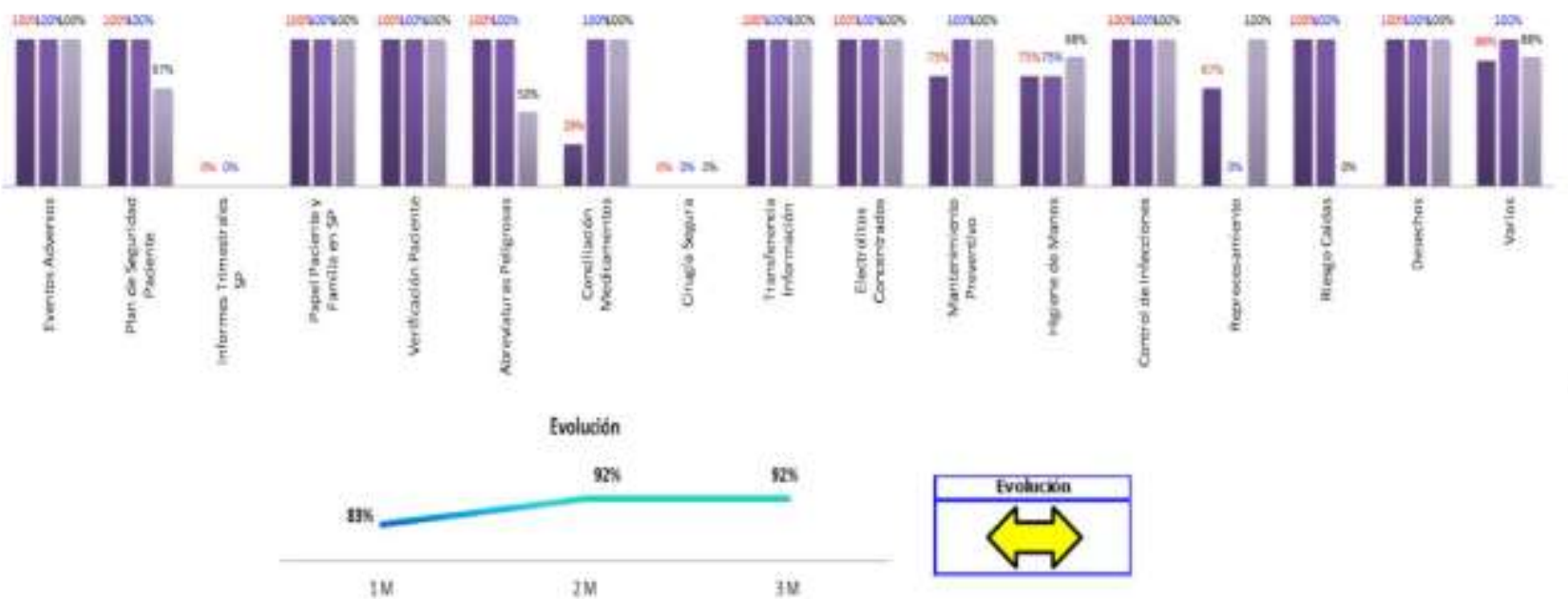

Fuente: Hospital Especialidades "José Carrasco Arteaga" Elaborado por: autores

Tabla Na 3 Tomada de Resultados de Evaluación. Plan de Seguridad del Paciente. Tercera Medición. Hospital José Carrasco Arteaga. Octubre 2018(14)

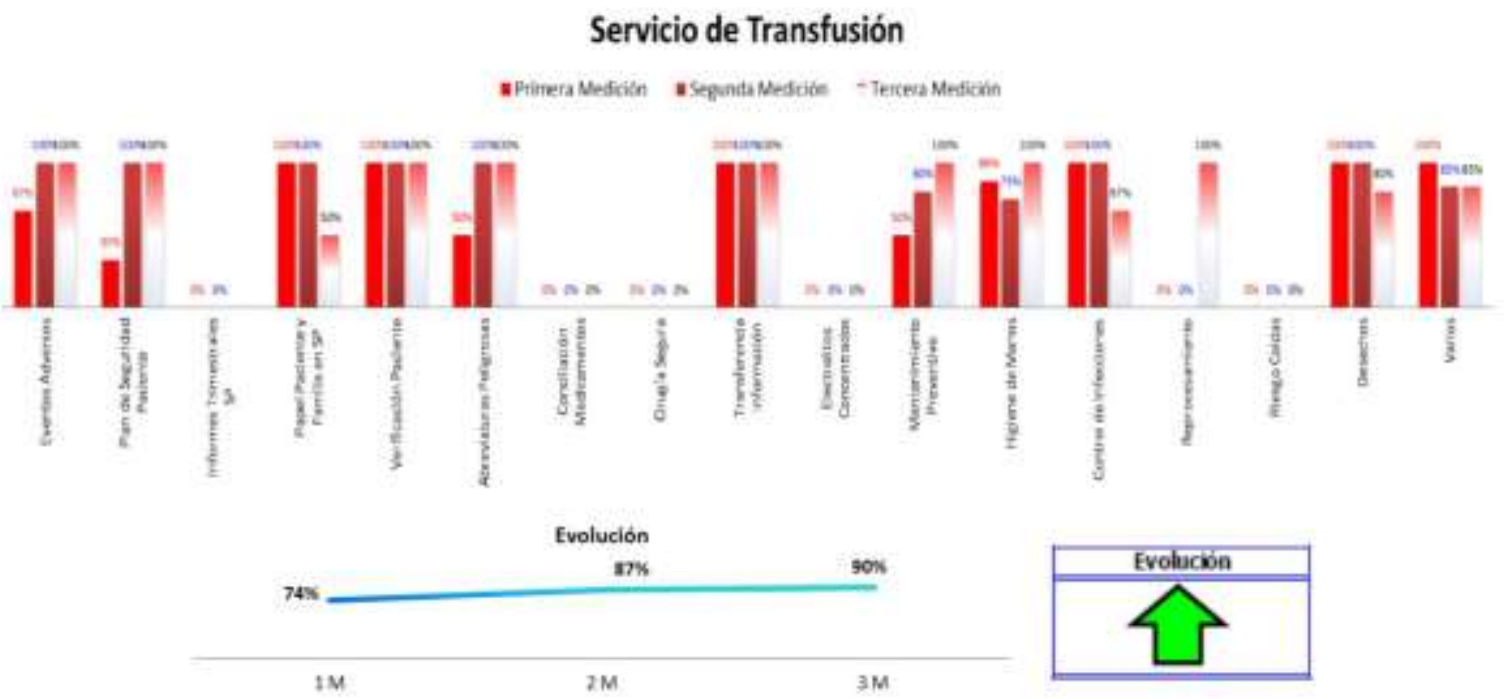

Fuente: Hospital Especialidades "José Carrasco Arteaga“

Elaborado por: autores 
Tabla $N^{\mathbf{a}} 4$ Tomada de Resultados de Evaluación. Plan de Seguridad del

Paciente. Tercera Medición. Hospital José Carrasco Arteaga. Octubre 2018(14)

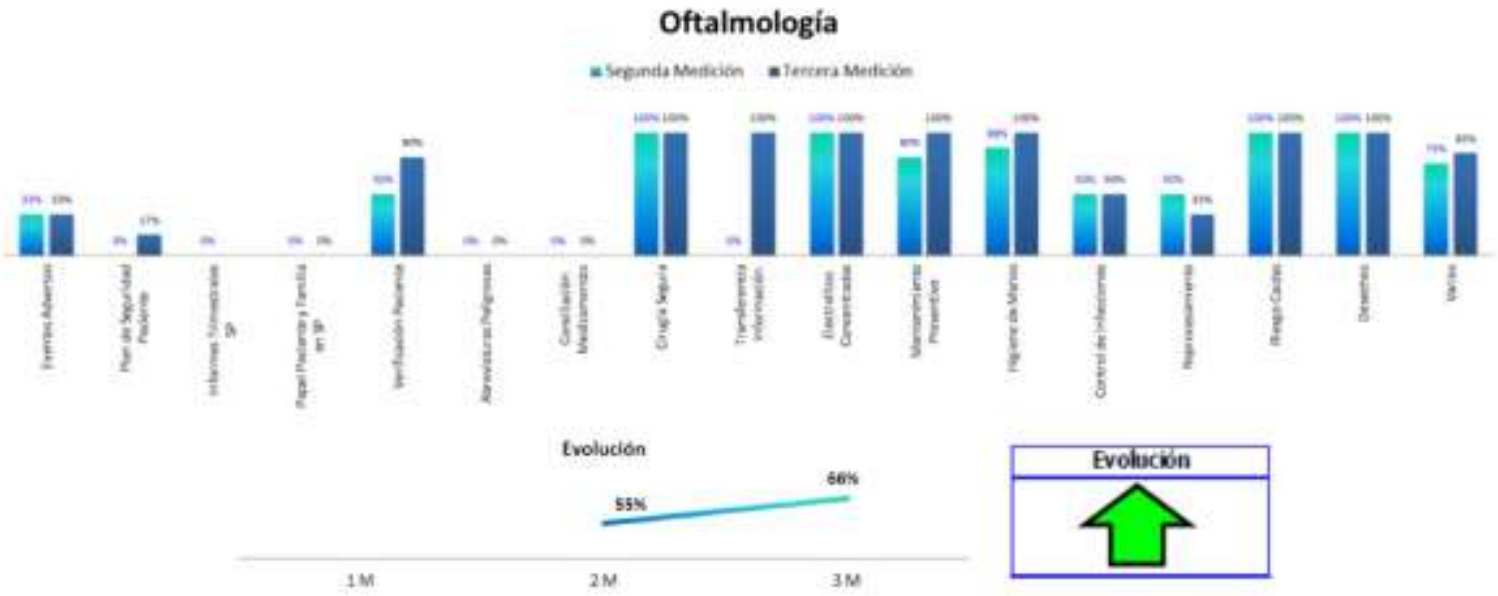

Tabla N 5 Tomada de Resultados de Evaluación. Plan de Seguridad del Paciente. Tercera Medición. Hospital José Carrasco Arteaga. Octubre 2018(14)

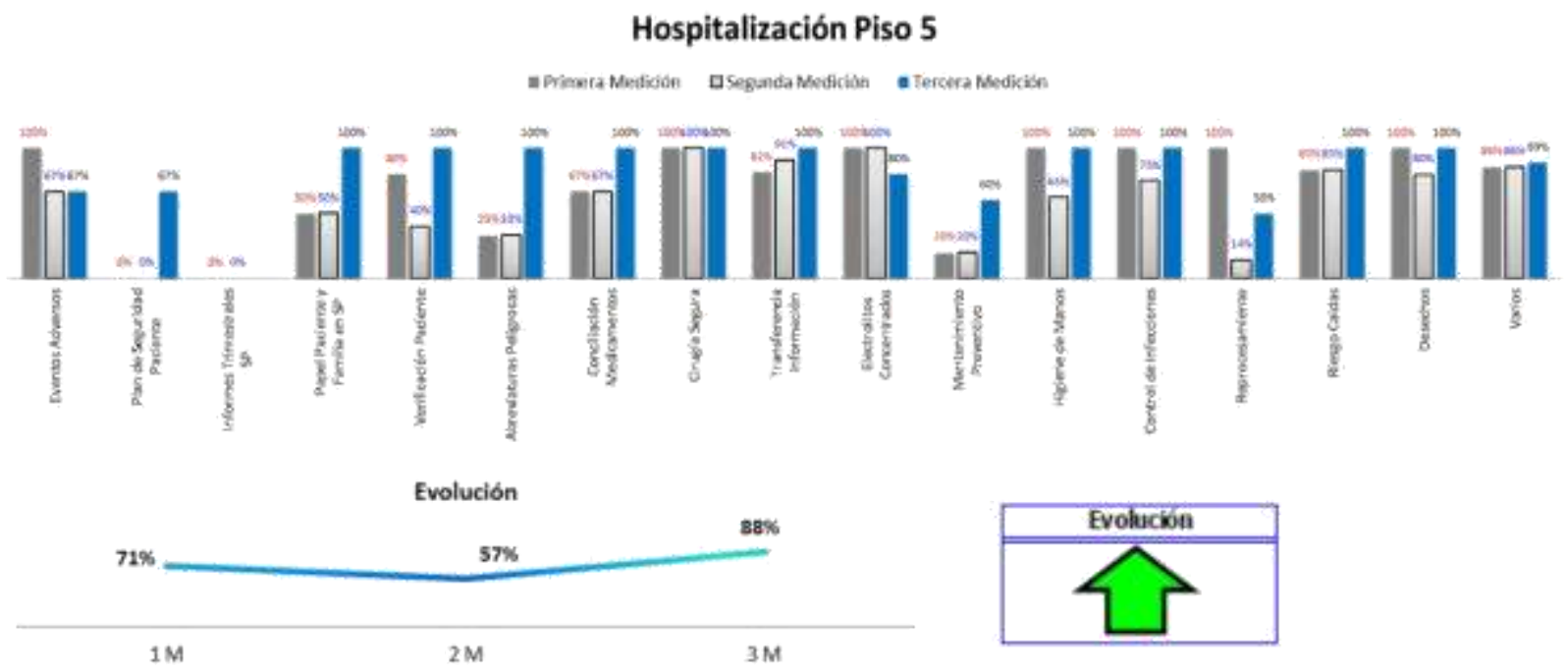

Fuente: Hospital Especialidades "José Carrasco Arteaga" Elaborado por: autores 
Tabla Na 6 Tomada de Resultados de Evaluación. Plan de Seguridad del Paciente. Tercera Medición. Hospital José Carrasco Arteaga. Octubre 2018(14)

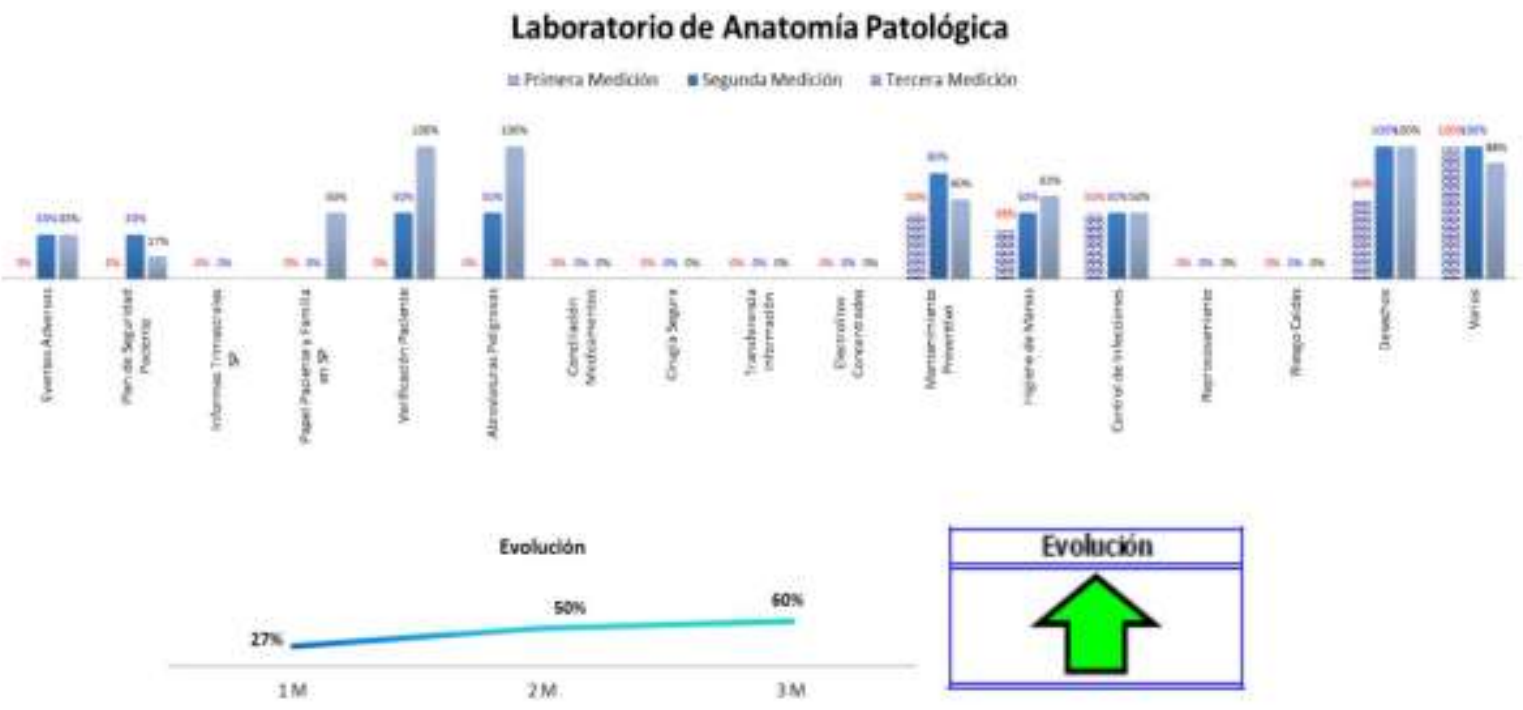

Fuente: Hospital Especialidades "José Carrasco Arteaga"

Elaborado por: autores

Tabla N 7 Tomada de Resultados de Evaluación. Plan de Seguridad del Paciente. Tercera Medición. Hospital José Carrasco Arteaga. Octubre 2018(14)

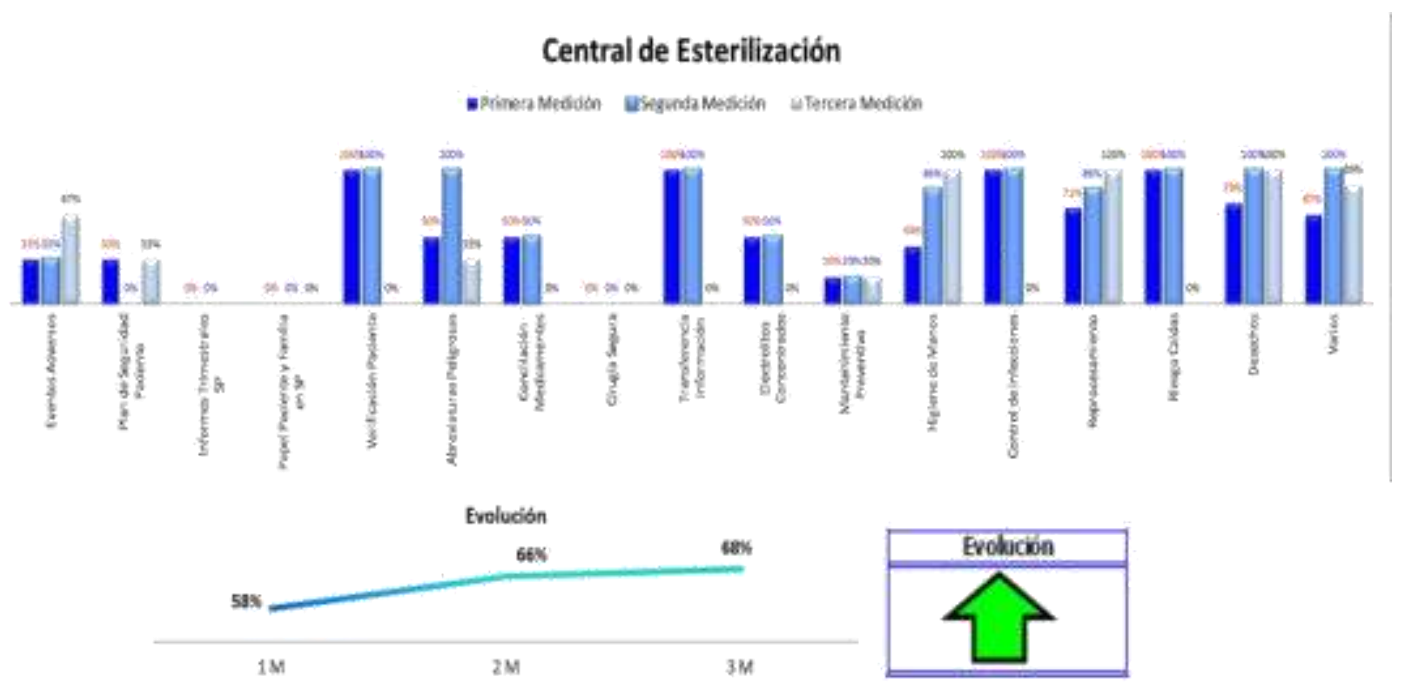

Fuente: Hospital Especialidades “José Carrasco Arteaga“" Elaborado por: autores 
Analizando los cuadros anteriores podemos encontrar que en la Tablas 3 - 4 y 5 encontramos una evolución favorable en la tercera medición del cumplimiento de prácticas seguras además que en las Tablas 6 y 7 también podemos encontrar una evolución favorable, pero a la vez no sobrepasa la línea de base (70\%).

\section{Conclusiones.}

- Según la Organización Mundial de Salud define a la Calidad Asistencial; como una atención sanitaria de alta calidad, es la que identifica las necesidades de salud educativa, preventiva, protectora y de los individuos o población de una manera total y concisa teniendo los recursos necesarios de una manera oportuna efectiva teniendo como resultado actual el conocimiento.

- En la actualidad se puso más énfasis en la calidad asistencial teniendo más fuerza en el mundo en 1994; la Joint Commission implantó el sistema de indicadores, siendo los principales medidores para la calidad a los ciudadanos y responsabilidad a los profesionales de la salud. Los indicadores para calidad asistencial son tres: estructura, proceso y resultado.

- El Hospital de Especialidades José Carrasco Arteaga se ha adaptado según las necesidades de los pacientes para el buen vivir, además que se implementa en Julio del 2018 un Plan de Seguridad del Paciente reforzando varios aspectos en la atención. (13)

- En el Hospital Especialidades José Carrasco Arteaga para las Prácticas Organizacionales requeridas basándose en la acreditación Internacional de Canadá en las prácticas seguras, así como la disponibilidad de los protocolos vigentes; valorando cada categoría y servicios de salud, a través de evaluaciones continuas en determinado tiempo de una manera aleatoria para mejorar cada día la Calidad Asistencial. (10)(11)

- Según la tercera evaluación del mes de octubre del 2018 se puede observar a través de cuadros comparativos de la primera, segunda y tercera medición de los diferentes servicios de prácticas seguras comparando la evolución de cada medición un incremento; además varios servicios no llegan a una línea base del $70 \%$ como oftalmología, central de esterilización y laboratorio de anatomía patológica dando como efecto la disminución del puntaje del cumplimiento para prácticas seguras.

- El 21\% de los servicios evaluados alcanzan el valor mínimo aceptable del $70 \%$ de cumplimiento de las prácticas organizacionales requeridas, lo cual refleja un incremento con respecto al desempeño obtenido en la medición previa.

- Como se puede observar en la tabla №2 los servicios de mayor nivel de cumplimiento son consulta externa 92\%, servicios de transfusión $90 \%$ y hospitalización 5to piso $88 \%$.

- Analizando la tabla $\mathrm{N}^{\circ} 2$ los tres servicios de menor puntaje de cumplimiento son laboratorio de anatomía patológica $60 \%$, oftalmología $66 \%$ y central de esterilización 68\%; sin embargo, hay que destacar que los tres servicios han tenido mejores resultados con respecto a mediciones previas.

- Comparando las tablas 3-4-5-6 y 7 observamos que el manejo de desechos obtiene el porcentaje más alto de cumplimiento a nivel global $98 \%$ por segunda vez consecutiva. El 50\% de servicios evaluados cumple al 100\% los parámetros 
en la higiene de manos, a comparación en que se obtuvo en $28 \%$ en la primera medición.

- En el estudio aleatorio que hemos planteado encontramos el 50\% de los servicios evaluados con la semaforización en escala verde siendo esto mayor al $85 \%$ y el $50 \%$ restante en escala roja menor al $70 \%$.

- Por tercera vez consecutiva una fuerte debilidad representa el conocimiento de las acciones que se están realizando sobre seguridad del paciente en la institución. Si bien es cierto se ha realizado acciones que permiten a los servicios conocer esta información, existiendo una deficiencia en mecanismos y estrategias de comunicación interna para generar mayor impacto.

- En el $45 \%$ de los casos los familiares, pacientes y usuarios desconocen su papel en fomento de la seguridad del paciente, siendo estos actores claves.

\section{Referencias bibliográficas.}

1.- Aula Esteve- sem FYC de calidad Asistencial y Seguridad del Paciente. 17 de julio del 2014.

2.- The principles of quality assurance.Qual Assur Health Care 1989; 1: 79-95.

3.- Rodríguez Pérez MP. Grande Arnesto M. Calidad asistencial: concepto, dimensiones y desarrollo operativo (Internet). Madrid Escuela Nacional de Sanidad; 2014.

4.- Alcazar Casanova Felix, Iglesias Alonso Fuencisla. Manual de Calidad Asistencial. Historia y Definición de los servicios Sanitarios. 2007.

5.- Torres Maritza. Modelo de Calidad de la atención médica de Avedis Donabedian. (Internet). 2011.

6.- Rodriguez Díaz Consuelo, Villena Ugarte Enrique. Formulación de Criterios. Manual de Calidad Asistencial. Historia y Definición de los servicios Sanitarios. 2007.

7.- Constitución de la República del Ecuador, acuerdo ministeriales № ${ }^{\circ}$ 00001124, El Ministerio de Salud Pública, Capítulo I, Artículo 1, Artículo 2, Artículo 3.

8.- Díaz - Regañon García - Alcala Rafael, Sánchez Álvarez Tomás, Díaz Avendaño Nuria, Hernández Campa Susana Manual de Calidad Asistencial. Calidad en el Sector Servicios 2007.

9.- García Ruiz José Antonio, Canca José Carlos, Jiménez Puente Alberto, Prueba Enrique. Calidad Asistencial. (Internet). 5ta Edición. 2015.

10.- Thompson Leslee Presidente y CEO. Acreditación Internacional de Canadá. 2 de septiembre del 2016. 
11.- Constitución de la República del Ecuador, Acuerdo Ministerial No 5186. Ministerio de Salud Pública. Artículo 5.

12.- Humet Carlos, Suñol Rosa. Revista de Calidad Asistencial. Edición Naa. 2001.

13.- Quintuña Alonso. Revista de 85 años de Historia. Edición 4. 2013.

14.- Espin Oscar. Resultados de Evaluación Seguridad del Paciente. Plan de Seguridad del Paciente. Tercera Medición. Coordinación General de Control de Calidad Hospital de Especialidades José Carrasco Arteaga.2018. 


\section{Para citar el artículo indexado.}

Torres Dávila, E., López Espinoza, M., Cáceres Manzano, V., \& Espín Maldonado, O. (2019). Estudio de procedimientos para mejorar el sistema de calidad en el hospital de especialidades "José Carrasco Arteaga" del Instituto Ecuatoriano de Seguridad Social Cuenca - Azuay. Ciencia Digital, 3(2.1), 56-69. https://doi.org/10.33262/cienciadigital.v3i2.1.430

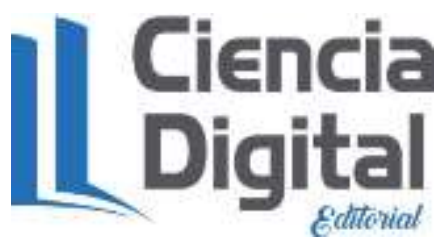

El artículo que se publica es de exclusiva responsabilidad de los autores y no necesariamente reflejan el pensamiento de la Revista Ciencia Digital.

El articulo queda en propiedad de la revista y, por tanto, su publicación parcial y/o total en otro medio tiene que ser autorizado por el director de la Revista Ciencia Digital.
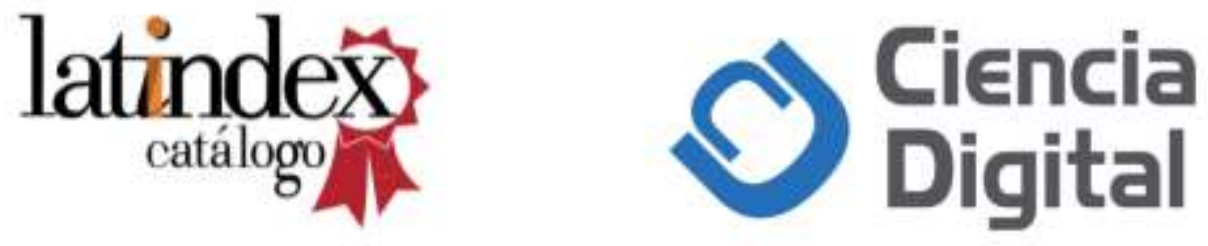